\title{
Themenschwerpunkt Überzeugungen von Lehrkräften im Kontext migrationsbezogener Heterogenität
}

\author{
Axinja Hachfeld • Marcus Syring
}

Online publiziert: 8 . September 2020

(C) Der/die Autor(en) 2020

In der Folge von Migrations- und Fluchtbewegungen stehen Lehrkräfte in deutschen Schulen sowie pädagogisches Personal in anderen Bildungseinrichtungen einer zunehmend kulturell heterogener werdenden Schülerschaft gegenüber. Aktuell hat fast jedes dritte schulpflichtige Kind Eltern, von denen mindestens ein Elternteil in einem anderen Land geboren wurde. Diese steigende migrationsbezogene, kulturelle Heterogenität erfordert von Lehrkräften sowie weiterem pädagogischen Personal professionelle Kompetenzen, welche neben Fach- und fachdidaktischem Wissen auch selbstregulative Fähigkeiten, Überzeugungen und Werthaltungen umfassen. Im Kontext migrationsbezogener Heterogenität wurden in den letzten Jahren vermehrt die migrationsbezogenen (,,kulturellen“) Überzeugungen von Lehrkräften untersucht, da Überzeugungen grundsätzlich eine handlungsleitende Funktion zugeschrieben wird. Der Blick der Forscherinnen und Forscher lag dabei zum einen auf der Beschreibung und der Operationalisierung der migrationsbezogenen Überzeugungen, zum anderen untersuchten sie die Zusammenhänge dieser Überzeugungen mit anderen Facetten professioneller Kompetenz sowie dem Unterrichtsverhalten von Lehrkräften.

Zusammenfassend zeigen die Forschungsarbeiten der letzten Jahrzehnte, dass sich die migrationsbezogenen Überzeugungen von Lehrkräften empirisch mithilfe von Fragebögen reliabel und valide erfassen lassen. Zudem stehen sie sowohl mit weiteren Facetten professioneller Kompetenzen, vor allem mit den motivationalen Orientierungen, als auch mit dem intendierten Unterrichtsverhalten der Lehrkräf-

Prof. Dr. A. Hachfeld $(\square)$

Pädagogische Hochschule Thurgau und Empirische Bildungsforschung, Universität Konstanz, Universitätsstraße 10/Fach 45, 78457 Konstanz, Deutschland

E-Mail: axinja.hachfeld@uni-konstanz.de

PD Dr. habil. M. Syring

Institut für Erziehungswissenschaft, Eberhard Karls Universität Tübingen,

Münzgasse 22, 72070 Tübingen, Deutschland

E-Mail: marcus.syring@uni-tuebingen.de 
te in Zusammenhang. Neuere Forschungsarbeiten betonen indessen die Wichtigkeit zwischen verschiedenen Dimensionen migrationsbezogener Überzeugungen zu unterscheiden. Je nach Konzeptualisierung lassen sich die empirischen Arbeiten zwei Gruppen zuordnen: Während die Arbeiten der einen Gruppe sich an IntergruppenIdeologien anschließen (siehe Kurucz, Hachfeld, Groeneveld, Roßbach \& Anders in diesem Heft), werden in den Arbeiten der anderen Gruppe (in Anlehnung an die Einstellungsforschung) die Überzeugungen in kognitive, affektive und verhaltensbezogene Komponenten unterteilt (siehe Gebauer \& McElvany in diesem Heft). Einen Einblick in aktuelle Forschungsarbeiten und -ergebnisse zu geben sowie Desiderate aufzuzeigen und Implikationen für die Praxis abzuleiten, ist das Ziel des vorliegenden Themenschwerpunktes. Vor dem Hintergrund der vielen aktuellen Forschungsprojekte in diesem Bereich ist anzunehmen, dass die hier versammelten Beiträge nur einen ersten Aufschlag und Ausschnitt bieten können. Weiterer Forschungsbedarf besteht beispielsweise hinsichtlich der Frage, welchen Einfluss die migrationsbezogenen Überzeugungen auf das tatsächliche Verhalten der Lehrkräfte ausüben (Dignath \& Kunter, in Vorbereitung). Im Weiteren gilt es empirisch zu klären, wie sich die migrationsbezogenen Überzeugungen auf die schulische Adaptation von Schülerinnen und Schülern (bspw. auf ihre schulische Eingebundenheit) sowie auf ihre Leistungsentwicklung auswirken (Schotte, Edele, Rjosk, Hachfeld \& Stanat, in Vorbereitung). Ebenfalls im Fokus aktueller Forschungen steht die kritische Auseinandersetzung mit den ,color-evasiven“ Überzeugungen (Civitillo, Juang \& Schachner, in Vorbereitung) und dem Integrationsverständnis von Lehrkräften (Kleen \& Glock, in Vorbereitung).

Der vorliegende Themenschwerpunkt stellt drei Arbeiten zu migrationsbezogenen Überzeugungen vor: In dem einführenden Stichwortartikel von Axinja Hachfeld und Marcus Syring wird das Forschungsfeld der migrationsbezogenen Überzeugungen systematisch mithilfe eines integrativen Reviews zusammengefasst und in seinem historischen Kontext verortet. Dabei blicken die Autorin und der Autor auf die letzten 20 Jahre der Forschung zu migrationsbezogenen Überzeugungen zurück (1998-2018) und ordnen die Forschungsbefunde entlang der vier Schwerpunkte: 1) Konzeptualisierung und Operationalisierung, 2) Auswirkungen auf das intendierte Verhalten der Lehrkraft und ihre professionellen Kompetenzen, 3) Prädiktoren migrationsbezogener Überzeugungen und 4) Auswirkungen auf die Schülerinnen und Schüler. Die Studien werden innerhalb der Schwerpunkte im Kontext der historisch-gesellschaftlichen Entwicklungen sowie der Entwicklungen in benachbarten Forschungsdisziplinen und in der Förderlandschaft betrachtet. Anhand dieses Status quo werden abschließend unter Berücksichtigung internationaler Perspektiven aufscheinende Forschungsdesiderate diskutiert.

In welchem Zusammenhang die migrationsbezogenen Überzeugungen von Lehrkräften mit der differenziellen Gestaltung des Unterrichts stehen, untersuchen $M i$ riam M. Gebauer und Nele McElvany in ihrem Beitrag. Im Fokus der Studie stehen Lehrkräfte an Gemeinschaftsschulen - eine Schulform, die sich per Definition auf eine stark heterogene Schülerschaft stützt und durch die explizite Wertschätzung individueller Förderung auszeichnet. Die migrationsbezogenen Überzeugungen operationalisieren die Autorinnen über die Einstellungen und differenzieren zwischen den Aspekten der Kosten, des Nutzens und der negativen Emotionen. Für die Unterrichts- 
gestaltung greifen die Autorinnen auf das Konzept der differentiellen Instruktion (Hall, 2002) zurück und unterscheiden die Phase der Instruktionsvorbereitung von den Phasen der Instruktionsdurchführung und der Instruktionsreflektion. Die Ergebnisse der Studie unterstützen die Wichtigkeit der Einstellungskomponente „Nutzen“ für die differentielle Instruktion, wobei sich die Zusammenhänge für die verschiedenen Phasen unterschieden. Die wahrgenommenen Kosten wiederum hatten keinen Einfluss auf die Phasen der differenziellen Instruktion. Interessanterweise ließen sich zudem sowohl schulformspezifische als auch genderspezifische Unterschiede finden. Ihre Ergebnisse diskutieren die Autorinnen demnach auch vor dem Hintergrund der Lehramtsausbildung.

Auch der Beitrag von Csaba Kurucz und Kolleginnen und Kollegen konzentriert sich im weiteren Sinne auf die Zusammenhänge der Überzeugungen mit der differenziellen Gestaltung von pädagogischen Situationen. Der Schwerpunkt dieser Studie liegt auf dem frühkindlichen Bereich und den multikulturellen Überzeugungen von 251 frühpädagogischen Fachkräften. Die Autoren und Autorinnen untersuchen in ihren Analysen, inwieweit multikulturelle Überzeugungen und diversitätsspezifische Selbstwirksamkeitserwartungen miteinander in Beziehung stehen und sich im zeitlichen Verlauf verändern. Darüber hinaus wird geprüft, in welchem Zusammenhang die beiden Konstrukte mit der wahrgenommenen Wichtigkeit verschiedener sprachpädagogischer Förderstrategien in Kindertageseinrichtungen stehen. In den Blick genommen wurden dabei alltagsintegrierte und additive Sprachförderansätze. Damit schlägt der dritte Beitrag nicht nur eine Brücke zu einer früheren Phase im Bildungsverlauf, sondern auch zum Bereich der Sprachförderung. Die Ergebnisse weisen auf einen signifikanten positiven Zusammenhang zwischen den multikulturellen Überzeugungen und der intendierten alltagsintegrierten sprachlichen Förderung hin. Da der Anteil von Kindern mit Migrationshintergrund in den jüngeren Altersstufen und im frühkindlichen Bereich besonders hoch ist, Tendenz steigend, scheinen künftig weitere Studien, die sich mit den Überzeugungen weiteren pädagogischen Personals beschäftigen, notwendig.

Funding Open Access funding provided by Projekt DEAL.

Open Access Dieser Artikel wird unter der Creative Commons Namensnennung 4.0 International Lizenz veröffentlicht, welche die Nutzung, Vervielfältigung, Bearbeitung, Verbreitung und Wiedergabe in jeglichem Medium und Format erlaubt, sofern Sie den/die ursprünglichen Autor(en) und die Quelle ordnungsgemäß nennen, einen Link zur Creative Commons Lizenz beifügen und angeben, ob Änderungen vorgenommen wurden.

Die in diesem Artikel enthaltenen Bilder und sonstiges Drittmaterial unterliegen ebenfalls der genannten Creative Commons Lizenz, sofern sich aus der Abbildungslegende nichts anderes ergibt. Sofern das betreffende Material nicht unter der genannten Creative Commons Lizenz steht und die betreffende Handlung nicht nach gesetzlichen Vorschriften erlaubt ist, ist für die oben aufgeführten Weiterverwendungen des Materials die Einwilligung des jeweiligen Rechteinhabers einzuholen.

Weitere Details zur Lizenz entnehmen Sie bitte der Lizenzinformation auf http://creativecommons.org/ licenses/by/4.0/deed.de. 